\title{
Increasing incidence of invasive pneumococcal disease and pneumonia despite improved vaccination uptake: surveillance in Hull and East Yorkshire, UK, 2002-2009
}

\author{
J. W. T. ELSTON ${ }^{*}$, A. SANTANIELLO-NEWTON ${ }^{2}$, J. A. MEIGH ${ }^{3}$, D. HARMER ${ }^{2}$, \\ V. ALLGAR ${ }^{4}$, T. ALLISON ${ }^{5}$, G. RICHARDSON ${ }^{6}$, R. MEIGH ${ }^{3}$, S. R. PALMER ${ }^{7}$ \\ AND G. BAR LOW 1 \\ ${ }^{1}$ Hull and East Yorkshire Hospitals NHS Trust, Department of Infection and Tropical Medicine, Hull, UK \\ ${ }^{2}$ Health Protection Agency, North Yorkshire and the Humber, UK \\ ${ }^{3}$ Hull and East Yorkshire Hospitals NHS Trust, Department of Medical Microbiology, Hull, UK \\ ${ }^{4}$ Hull York Medical School, Department of Medical Statistics, York, UK \\ ${ }^{5}$ NHS East Riding of Yorkshire and East Riding of Yorkshire Council, East Riding of Yorkshire, UK \\ ${ }^{6}$ University of York, Centre for Health Economics, York, UK \\ ${ }^{7}$ Cardiff University, Department of Primary Care and Public Health, Cardiff, UK
}

(Accepted 26 August 2011; first published online 1 November 2011)

\section{SUMMARY}

Introduction of pneumococcal polysaccharide (PPV23) and conjugate vaccine (PCV7) programmes were expected to change the epidemiology of invasive pneumococcal disease (IPD) and pneumonia in the UK. We describe the epidemiology of IPD and hospitalization with pneumonia using high-quality surveillance data over an 8-year period, 2002-2009. Although PPV23 uptake increased from $49 \%$ to $70 \%$ and PCV7 uptake reached $98 \%$ by 2009 , the overall incidence of IPD increased from $11 \cdot 8 / 100000$ to $16 \cdot 4 / 100000(P=0 \cdot 13)$, and the incidence of hospitalization with pneumonia increased from $143 / 100000$ to $207 / 100000(P<0 \cdot 001)$. Although a reduction in the proportion of IPD caused by PCV7 serotypes was observed, concurrent increases in PPV23 and non-vaccine serotype IPD contributed to an increased IPD burden overall. Marked inequalities in the geographical distribution of disease were observed. Existing vaccination programmes have, so far, not been sufficient to address an increasing burden of pneumococcal disease in our locality.

Key words: Epidemiology, pneumococcal infection, vaccines.

\section{INTRODUCTION}

Streptococcus pneumoniae is a leading cause of morbidity and mortality from community-acquired pneumonia (CAP) worldwide, and can cause invasive disease, for example, bacteraemia, meningitis, and

* Author for correspondence: Dr J. W. T. Elston, Department of Infection and Tropical Medicine, Main Administration block, Castle Hill Hospital, Cottingham, East Yorkshire, HU16 5JQ, UK. (Email: jameselston2003@yahoo.co.uk) septic arthritis [1,2]. There are currently two pneumococcal vaccines used in the UK, where all vaccine programmes are publically funded without cost to the user at the point of care. The pneumococcal polysaccharide vaccine (PPV23), which contains antigens from 23 serotypes of $S$. pneumoniae, was introduced in 1992 for high-risk groups [those with asplenia or dysfunction of the spleen, chronic respiratory, heart, renal or liver disease, diabetes (requiring insulin or oral hypoglycaemic drugs), immunosuppression, 
individuals with cochlear implants, and individuals with cerebrospinal fluid (CSF) leaks]. The PPV23 programme was expanded to cover all persons aged $\geqslant 65$ years in April 2005 (phased introduction with vaccination initially recommended to all those aged $>80$ years in August 2003 and then to those aged $>75$ years in April 2004). The pneumococcal conjugate vaccine $(\mathrm{PCV})$, which previously contained antigens from seven serotypes (PCV7) and since 2010 has covered 13 serotypes (PCV13), was introduced for those aged $<2$ years at a higher risk of pneumococcal infection in 2002, and was subsequently included in the routine childhood immunization programme in September 2006.

There is controversy about the effectiveness of PPV23 in older adults [3-6]. Only one small randomized controlled trial has demonstrated protective efficacy of PPV23 against pneumonia [7]. Evidence from observational studies has suggested that PPV23 is effective in reducing the incidence of invasive pneumococcal disease (IPD) (protective efficacy of about $45 \%$ ) [8-10], but not pneumonia or mortality, although the validity of this evidence has been questioned [6]. There is no controversy regarding PCV7, which has been demonstrated to be effective in reducing vaccine serotype pneumococcal disease in children in the USA [11]. Furthermore, a reduction in IPD in all age groups in the USA has been attributed to herd immunity following the introduction of $\mathrm{PCV} 7$ into routine childhood immunization programmes $[12,13]$. PCV7 has subsequently been introduced across Europe, where early studies have demonstrated a reduction in IPD rates in children and adults [14-17]. However, vaccine serotype coverage by PCV7 in the USA $(>80 \%)$ was greater than that in Europe $(60-70 \%)$ at the time of its introduction and a greater increase in non-PCV serotype disease has subsequently been recorded in European countries [14-18]. Because it is not guaranteed that the effect of vaccination will be the same in all populations, even within the same country, high-quality surveillance data is essential at both local and national levels to monitor the epidemiology of pneumococcal disease.

The population of Hull ( 264500) has a much higher prevalence of comorbid illness and unhealthy behaviour, such as smoking, and a shorter life expectancy than the average for England, and is known to be more deprived with over $50 \%$ of the population living within the most deprived national quintile [19, 20]. The population of the East Riding of Yorkshire (ERoY) ( 346000), in comparison, is relatively affluent with a longer life expectancy and better health indicators than averages for England [19, 21]. In both Hull and ERoY, PPV23 has been recommended for all those aged $\geqslant 65$ years since 2002, anticipating the national recommendation by 3 years. The local Health Protection Unit (HPU), in contrast to many other areas of the UK, has been actively collecting patient-level surveillance data from primary-care General Practitioners (GPs) on PPV23 vaccination uptake since its introduction.

The purpose of this study was to describe the epidemiology of IPD and pneumonia in Hull and ERoY over an 8-year period during which PPV23 and PCV7 vaccination programmes were introduced. Our primary hypothesis was that a reduction in the incidence of IPD and pneumonia in the target groups for vaccination would have occurred during the study period. Secondary hypotheses were that: a reduction in the incidence of IPD and pneumonia would have occurred in other age groups as a result of herd immunity, particularly in relation to PCV7 introduction; vaccine serotype IPD would have decreased; and that there would be an association between the incidence of IPD and the degree of deprivation within a locality.

\section{METHODS}

\section{Surveillance and data collection}

The computer databases of the microbiology laboratories of Hull and East Yorkshire Hospitals (HEY) NHS Trust and of hospitals from three NHS Trusts bordering Hull and ERoY (comprising eight hospitals in total that routinely receive admissions of individuals resident in Hull and ERoY) (Supplementary Table S1, available online), were retrospectively interrogated in order to identify pneumococcal isolates cultured from samples retrieved from normally sterile body sites (blood, CSF, and joint, pleural or ascitic fluids), from 1 January 2002 to 31 December 2009. Information from all four Trust microbiology laboratories was obtained for the complete time period 2002-2009 with the exception of one, North Lincolnshire and Goole (NLG) Hospitals NHS Foundation Trust, where data for the years 2002 and 2003 were unavailable. The main NLG hospital concerned lies outside the boundaries of Hull and ERoY and, in relation to this study, serves a small catchment area of just one postcode district (Goole, DN14). In order to account for the missing data, the population 
numbers for that postcode for 2002 and 2003 were subtracted from all the population denominators used in the calculation of incidence rates. In addition, positive pneumococcal PCR results for residents of Hull and ERoY were retrospectively identified from the reports received from supra-regional testing laboratories held by the HPU. Individuals were classified as a case of IPD if $S$. pneumoniae was cultured from a normally sterile body site and/or pneumococcal DNA was detected by PCR of blood and/or CSF. Demographic details of cases (including postcode district) were obtained from the Trust microbiology and/or pathology databases. Only individuals resident in Hull and ERoY, defined by the postcode of residence lying within the recognized geographical boundaries of the Hull and ERoY Primary Care Trusts (PCTs), were included. Information concerning the origin of pneumococcal isolates cultured from samples received within the first $24 \mathrm{~h}$ of admission, and the serotype of isolates (tested by the Respiratory and Systemic Infection Laboratory, Colindale, London, UK) were recorded. In order to avoid the same patient episode being included more than once, further subsequent isolates from specimens taken within 30 days of the index isolate(s) were excluded.

In order to make an assessment of the contribution of changing laboratory and/or clinical (sampling) practice in relation to the diagnosis of IPD, information on the number of blood cultures submitted to microbiology laboratories and changes in laboratory practices were obtained. Numbers of hospital admissions were also obtained from hospital trust information services in order to calculate the incidence of blood culture sampling.

For the years $2005 / 2006$ to $2009 / 2010$, information on population vaccination uptake was derived from the results of PPV uptake surveys, performed and published by the Health Protection Agency (HPA), concerning the populations covered by Hull and ERoY PCTs, using the same geographical boundaries as defined above [22]. For the years 2001/2002 to $2004 / 2005$, in which HPA surveys were not performed, vaccination uptake was calculated using data from the HPU PPV vaccination surveillance database. PPV vaccination status of IPD cases aged $\geqslant 65$ years was ascertained by matching the identity of IPD cases with individuals in the HPU vaccination surveillance database. Both the national HPA and the local HPU datasets provided population numbers vaccinated (and not vaccinated) by year, which were used as denominator populations (person-years) in the calculation of PPV23 effectiveness as detailed below. Date of death (if applicable) of IPD cases was recorded from the National Health Service Spine Summary Care database.

Hull and ERoY PCTs provided data on the number of hospital admissions of residents of Hull and ERoY with a diagnosis of pneumonia (2002-2009), which was established by searching the Healthcare Purchaser System database (containing information from the Secondary Uses Service, the national central repository for healthcare data) for the following ICD-10 codes in the primary diagnostic field: J18.0 bronchopneumonia, J18.9 pneumonia (unspecified), J13X pneumococcal pneumonia, J18.1 lobar pneumonia and J15X bacterial pneumonia not elsewhere classified; residence in Hull and ERoY was defined by residential postcode and/or GP registration lying within the PCT geographical boundaries outlined above. As an unrelated 'observational control' (for data validation purposes), the numbers of admissions of residents with a primary diagnosis of fractured neck of femur over the same time period were established by the same method for the following ICD-10 codes: S720 fracture of neck of femur, S721 pertrochanteric fracture, S722 subtrochanteric fracture. To replicate the method used to identify IPD cases and compare the incidence of IPD with another community-acquired infectious disease for which a vaccine is not available, the number of confirmed invasive group $\mathrm{B}$ meningococcal infections in residents of Hull and ERoY from 2002 to 2009, whose samples were obtained from the same hospitals, was determined from records held at the HPU. [Invasive group $\mathrm{B}$ meningococcal infections (diagnosed when group B meningococcus is cultured or diagnosed by PCR from a sample retrieved from a sterile body site, and there are clinical signs and symptoms indicative of infection), are subject to statutory notification to HPUs in the UK.]

\section{Statistical analyses and hypotheses testing}

Descriptive statistics are presented as numbers and percentages. Crude incidence rates were calculated using mid-year population estimates for Hull and ERoY published by the Office for National Statistics (ONS) [19]. Age- and sex-specific incidence rates were calculated. The $95 \%$ confidence interval (CI) formula used is based on the Poisson distribution.

Poisson regression was undertaken to compare the incidence rates annually; and was also undertaken 
to compare incidence rates within age groups and by gender in order to detect changes in incidence (as used in a previous UK IPD study) [10]. $\chi^{2}$ tests were used to compare the proportions of IPD cases caused by vaccine and non-vaccine serotypes. PPV23 effectiveness was estimated using the screening method:

$100 \times \frac{\text { IR in unvaccinated group-IR in vaccinated group }}{\text { IR in unvaccinated group }}$,

where IR = incidence rate [23].

In order to make comparisons, incidence density rates were calculated separately for the populations of Hull and ERoY, for the entire study period using ONS population estimates as the denominator. Incidence density rates were also calculated by postcode district separately for Hull and ERoY for the entire study period using resident population estimates (residence defined by postcode, as above) supplied by Hull and ERoY PCTs from the primary care, general practice (GP), patient registration file as the denominator. Incidence density rates were directly age-standardized to the European Standard Population and $95 \%$ CIs were calculated according to the preferred method of the Association of Public Health Observatories (APHO) (utilizing preformulated APHO spreadsheet tools), using Byar's approximation (for numerators above 389) or computed from the Poisson distribution (for numerators $\leqslant 389$ ) [24]. To compare incidence rates between Hull and ERoY and between postcode districts, Poisson regression was undertaken. A Mann-Whitney test was undertaken to compare age at death between Hull and ERoY and $\chi^{2}$ tests were used to compare casefatality. A population-weighted mean deprivation score [index of multiple deprivation (IMD) 2007] was calculated for each postcode district and was supplied by the PCTs [25]. Spearman's rank correlations were calculated to explore the relationship between deprivation and incidence of IPD which was assumed to be nonlinear in nature.

To explore the relationship between numbers of blood cultures submitted and IPD incidence, Spearman's rank correlations were calculated. Poisson regression was undertaken to compare blood culturesampling incidence rates between annual data in order to assess any potential changes in sampling practices. In the same manner as for IPD, Poisson regression was undertaken to compare incidence rates for fractured neck of femur and for invasive group B meningococcal disease.
A $P$ value of $<0.05$ was considered to indicate statistical significance. No adjustments were made for multiple testing in keeping with the recommendations of Perneger, who stated that 'simply describing what tests of significance have been performed, and why, is generally the best way of dealing with multiple comparison' [26]. All data were recorded and analysed using PASW Statistics v. 18 (IBM, USA). Data and information sources are summarized in Supplementary Table $\mathrm{S} 2$ (online).

Advice was sought from the National Research Ethics Service (NRES) prior to study commencement; this work was classified as surveillance and did not require formal review by an ethics committee.

\section{RESULTS}

\section{Study sample}

Of the 653 patients with IPD (730 positive microbiological samples), $350(53.6 \%)$ were male; their median age was 68 years $(55.7 \%$ aged $\geqslant 65$ years, age range $0-102$ years). The age and gender distribution of IPD cases is shown in Figure 1. The ratio of male to female IPD cases was $1 \cdot 16$. There were 668 $(91.5 \%)$ pneumococcal isolates cultured from blood, $17(2 \cdot 3 \%)$ from CSF, $17(2 \cdot 3 \%)$ from pleural fluid, eight $(1 \cdot 1 \%)$ from synovial fluid and three $(0 \cdot 4 \%)$ from ascitic fluid; PCR results indicative of pneumococcal infection were retrieved for 16 samples: 10 $(1.4 \%)$ from CSF and six $(0.8 \%)$ from blood.

In total, $561(85.9 \%)$ of the 653 individuals had presented to the three hospitals of HEY Hospitals NHS Trust, $49(7.5 \%)$ to the two hospitals of Scarborough and North East Yorkshire (NEY) NHS Trust, $27(4 \cdot 1 \%)$ to York Teaching Hospital (YTH), and $16(2.5 \%)$ to the two hospitals of North Lincolnshire and Goole (NLG) Hospitals NHS Foundation Trust.

\section{Incidence of IPD}

The number of confirmed cases of IPD increased from 65 in 2002 to 99 in 2009, corresponding to a rise in overall incidence of IPD from $11.8(95 \%$ CI 9.14-15.1) to $16 \cdot 4(95 \%$ CI $12 \cdot 4-18 \cdot 9)$ per 100000 (Table 1) (Poisson regression, $P=0 \cdot 13$ ). The incidence of IPD by age range is shown in Figure 2. The incidence of IPD in those aged $\geqslant 65$ years (the target group for PPV23) increased from 40.0 (95\% CI $28 \cdot 3-54 \cdot 9)$ to $45 \cdot 5(95 \%$ CI $33 \cdot 5-60 \cdot 3)$ per 100000 


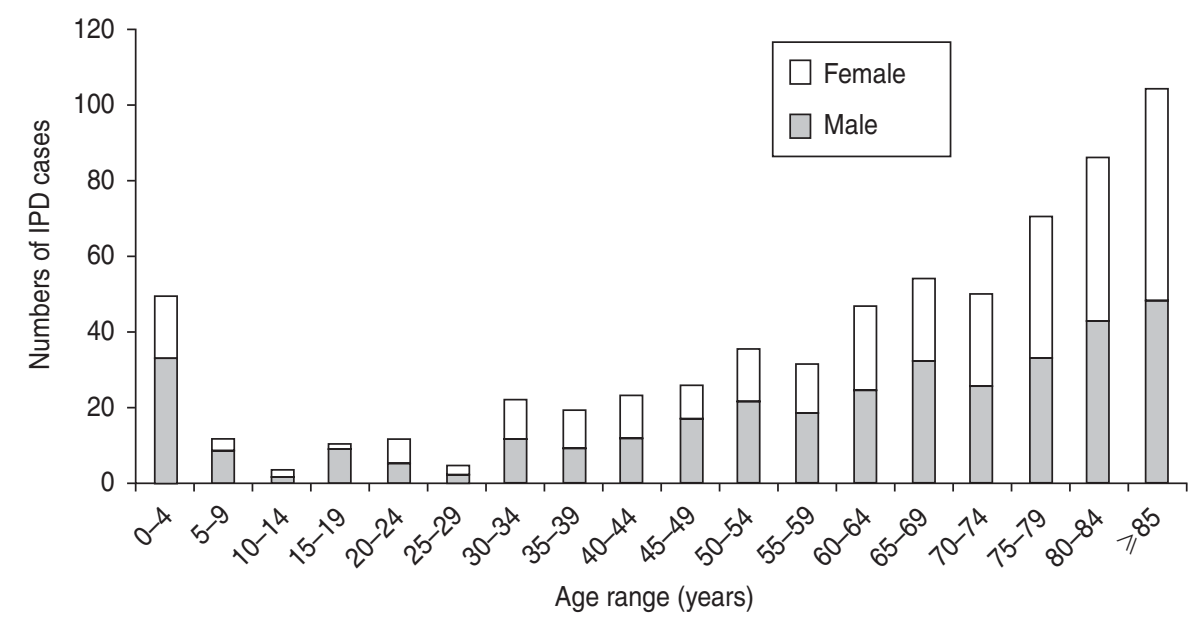

Fig. 1. Age and gender of invasive pneumococcal disease (IPD) cases.

from 2002 to 2009 (Poisson regression, $P=0.69$ ). In contrast, the incidence of IPD in those aged 15-64 years significantly increased from $5.0 \quad(95 \%$ CI $3 \cdot 0-8 \cdot 0)$ to $11 \cdot 2(95 \%$ CI $8 \cdot 2-15 \cdot 0)$ per 100000 (Poisson regression, $P=0 \cdot 02$ ) over the study period. There was no significant change in the incidence of IPD in those aged 5-14 years (Poisson regression, $P=0.91$ ); nor in those aged $<5$ years (includes the target group for PCV7) over the study period (Poisson regression, $P=0.54$ ) or post-PCV7 introduction (Poisson regression, $P=0 \cdot 34$ ).

The incidence rates for IPD in Hull and ERoY are compared to published national rates and those recently published for the population of Oxfordshire, England in Table 1.

\section{Vaccination uptake}

Over the same period, vaccination uptake for PPV in the $\geqslant 65$ years age group increased from $49 \%$ (95\% CI $48 \cdot 6-49 \cdot 3)$ to $70 \%(95 \%$ CI 69.5-70.0) and uptake of PCV7 in those aged $<2$ years had reached $98 \%$ (95\% CI 95.3-100·1) by 2009 (Fig. 3).

\section{Blood culture sampling, laboratory methods and antimicrobial susceptibilities}

The number of blood cultures received by HEY Hospitals NHS Trust (where $85.9 \%$ of the IPD cases were diagnosed) each year and the incidence of IPD is shown in Figure 4. There was no significant correlation between the numbers of blood cultures submitted and the incidence of IPD (Spearman's rank correlations, $\left.r_{s}=0.48, P=0.23\right)$. The incidence of blood culture sampling in those admitted to HEY hospitals actually decreased over the study period from 103 (95\% CI 101-104) to 91 (95\% CI 89-92) per 1000 admissions (Poisson regression, $P<0 \cdot 001$ ). In addition there were no reported major changes in laboratory methodology in relation to the diagnosis of IPD or pneumonia over the study period. Antimicrobial susceptibilities were available for all 714 culture isolates. Resistance to penicillin occurred in eight $(1 \cdot 1 \%)$ isolates and resistance to macrolides in $63(8.8 \%)$; resistance was stable over the study period.

\section{Mortality following IPD}

A total of $141(21.6 \%)$ IPD cases had died within 30 days of the invasive pneumococcal isolate. This included five deaths in those aged $<5$ years $(10 \cdot 2 \%$ of IPD cases aged $<5$ years), zero in those aged 5-14 years, 30 in those aged $15-64$ years $(13 \cdot 3 \%$ of cases aged $15-64$ years), and 106 in those aged $\geqslant 65$ years ( $29 \cdot 1 \%$ of cases aged $\geqslant 65$ years). Within 1 year 240 $(36.8 \%)$ cases had died, of which $50(20.8 \%)$ were aged $15-64$ years and $185(77 \cdot 1 \%)$ were aged $\geqslant 65$ years (there were no additional deaths between 30 days and 1 year in those aged $<5$ years). The casefatality rate within 30 days was non-significantly higher in men $(24 \cdot 3 \%$ vs. $18 \cdot 5 \%)\left(\chi^{2}, P=0 \cdot 07\right)$.

\section{Pneumococcal serotypes}

A serotype result was available for $478(75 \%)$ of the 639 culture-positive IPD cases. The number and proportion of IPD cases for which a serotype result was available increased over time (Table 2). Overall, 34 distinct pneumococcal serotypes were detected. Of the 
Table 1. Number of cases, incidence and mortality of invasive pneumococcal disease per 100000 by year

\begin{tabular}{lllllc}
\hline \hline Year & $\begin{array}{l}\text { Number of } \\
\text { cases }\end{array}$ & $\begin{array}{l}\text { Incidence, } \\
\text { Hull and } \\
\text { ERoY }\end{array}$ & $\begin{array}{l}\text { 30-day } \\
\text { mortality }\end{array}$ & $\begin{array}{l}\text { Incidence } \\
\text { Oxfordshire, } \\
\text { England* [17] }\end{array}$ & $\begin{array}{l}\text { Incidence } \\
\text { England } \\
\text { and Wales* [27] }\end{array}$ \\
\hline 2002 & 65 & $11 \cdot 8$ & $14(22 \%)$ & $8 \cdot 9$ & $9 \cdot 1$ \\
2003 & 60 & $10 \cdot 9$ & $8(13 \%)$ & $10 \cdot 3$ & $10 \cdot 1$ \\
2004 & 74 & $12 \cdot 6$ & $12(16 \%)$ & $8 \cdot 8$ & $10 \cdot 8$ \\
2005 & 90 & $15 \cdot 3$ & $20(22 \%)$ & $7 \cdot 9$ & $11 \cdot 8$ \\
2006 & 81 & $13 \cdot 8$ & $24(30 \%)$ & $8 \cdot 4$ & $11 \cdot 9$ \\
2007 & 91 & $15 \cdot 4$ & $19(21 \%)$ & $8 \cdot 7$ & n.a. \\
2008 & 93 & $15 \cdot 5$ & $27(29 \%)$ & $8 \cdot 1$ & n.a. \\
2009 & 99 & $16 \cdot 4$ & $17(17 \%)$ & $7 \cdot 1$ & n.a. \\
\hline \hline
\end{tabular}

ERoY, East Riding of Yorkshire; n.a., not available.

* Rates were measured July-June, therefore 2002 refers to rates of 2001/2002, 2003 to 2002/2003 and so on; rates for 2007-2009 for England and Wales were not available.

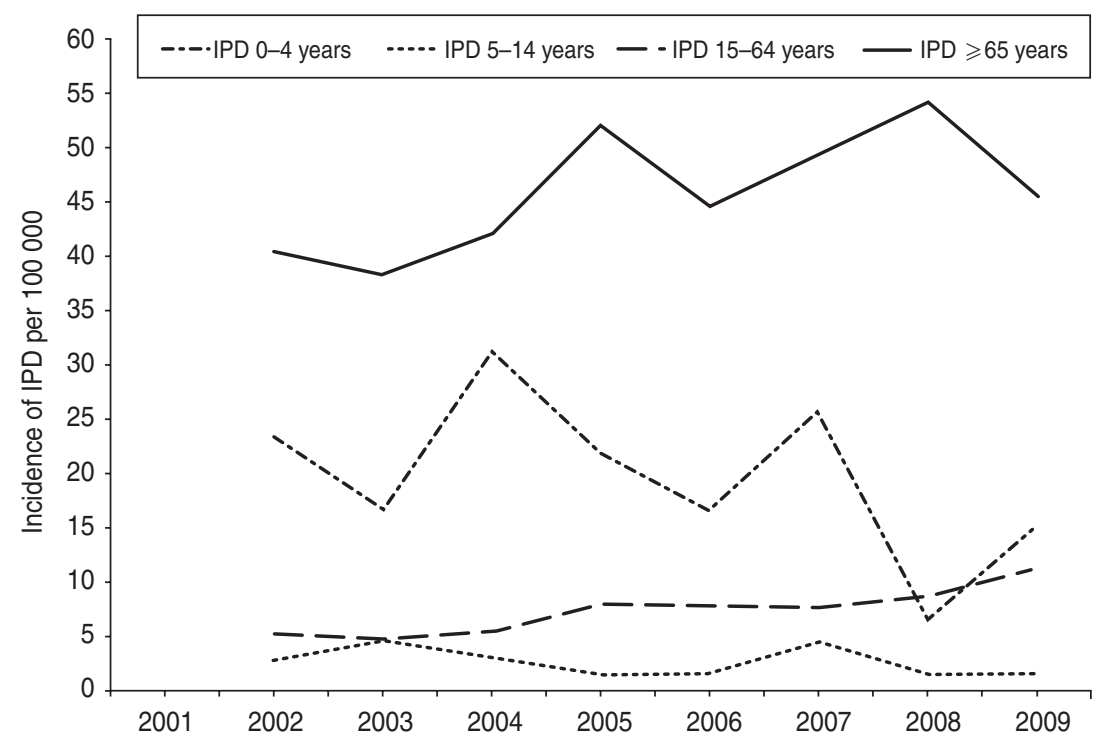

Fig. 2. Incidence of invasive pneumococcal disease (IPD) by age range.

isolates $426(89 \%)$ were PPV23 serotypes and 157 $(33 \%)$ were PCV7 serotypes. The proportion of IPD cases caused by serotypes covered by both PPV23 and PCV7 (the PCV7 serotypes) did not change significantly prior to PCV7 introduction (range 39-61\%, $\left.\chi^{2}, P=0 \cdot 34\right)$; however, post-PCV7 introduction fell sharply (from a baseline of $52 \%$ to $12 \%, \chi^{2}$, $P<0 \cdot 001)$, and the proportion of IPD cases caused by PPV23 serotypes increased post-PCV7 (from 39\% to $\left.69 \%, \chi^{2}, P<0 \cdot 05\right)$. The proportion of IPD cases caused by non-vaccine serotypes appeared to increase post-PCV7 introduction (from $9 \%$ to $19 \%$ ), although this was non-significant $\left(\chi^{2}, P=0 \cdot 26\right)$ (Table 2$)$.

\section{Vaccine effectiveness}

Of the 364 IPD cases aged $\geqslant 65$ years, $212(58 \%)$ were known to have received PPV23 prior to their episode of IPD. The median time from vaccination with PPV23 to IPD episode was 4 years and 2 months (range 2 months to 13 years). Serotype information was available for $164(77 \%)$ of the previously vaccinated cases; 138 (84\%) of these had infections caused by PPV23 serotypes and were therefore considered 'vaccine failures'. Overall incidence in the $\geqslant 65$ years age group known to have previously received PPV23 was $41 \cdot 5(95 \%$ CI $36 \cdot 1-47 \cdot 5)$ per 100000 person-years 


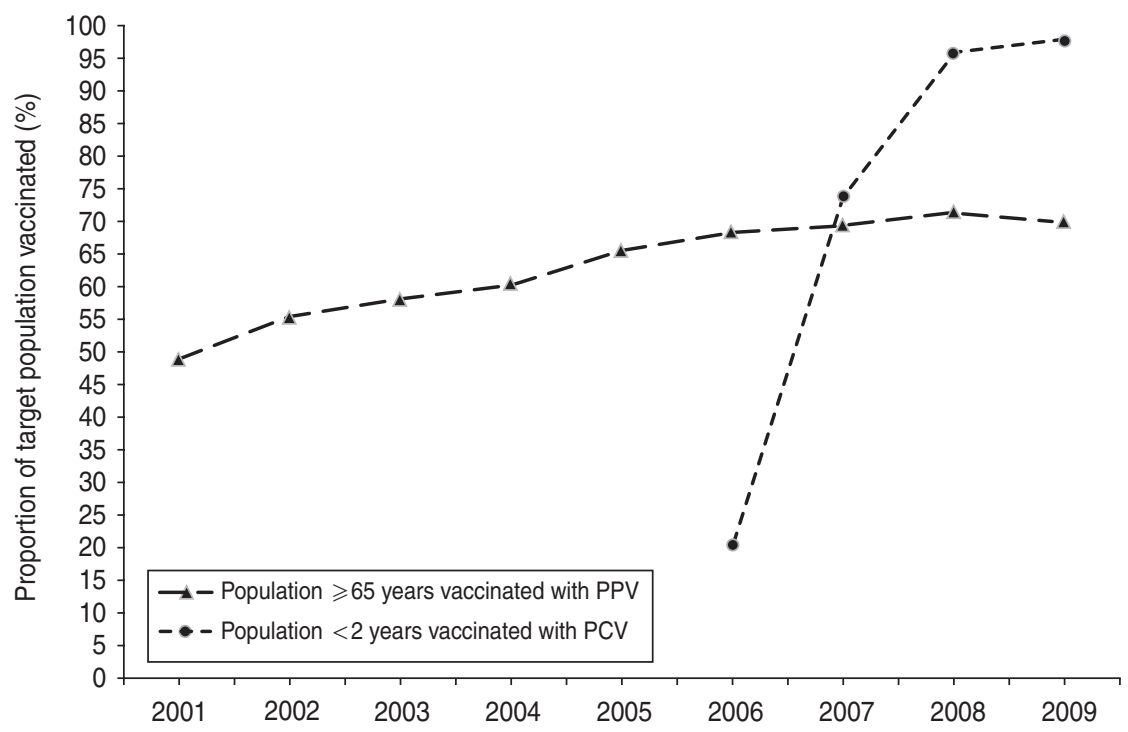

Fig. 3. Population vaccine uptake. (Vaccination uptake is calculated from 1 April to 31 March.) Data for 2001 represents uptake from 1 April 2001 to 31 March 2002, and so on.

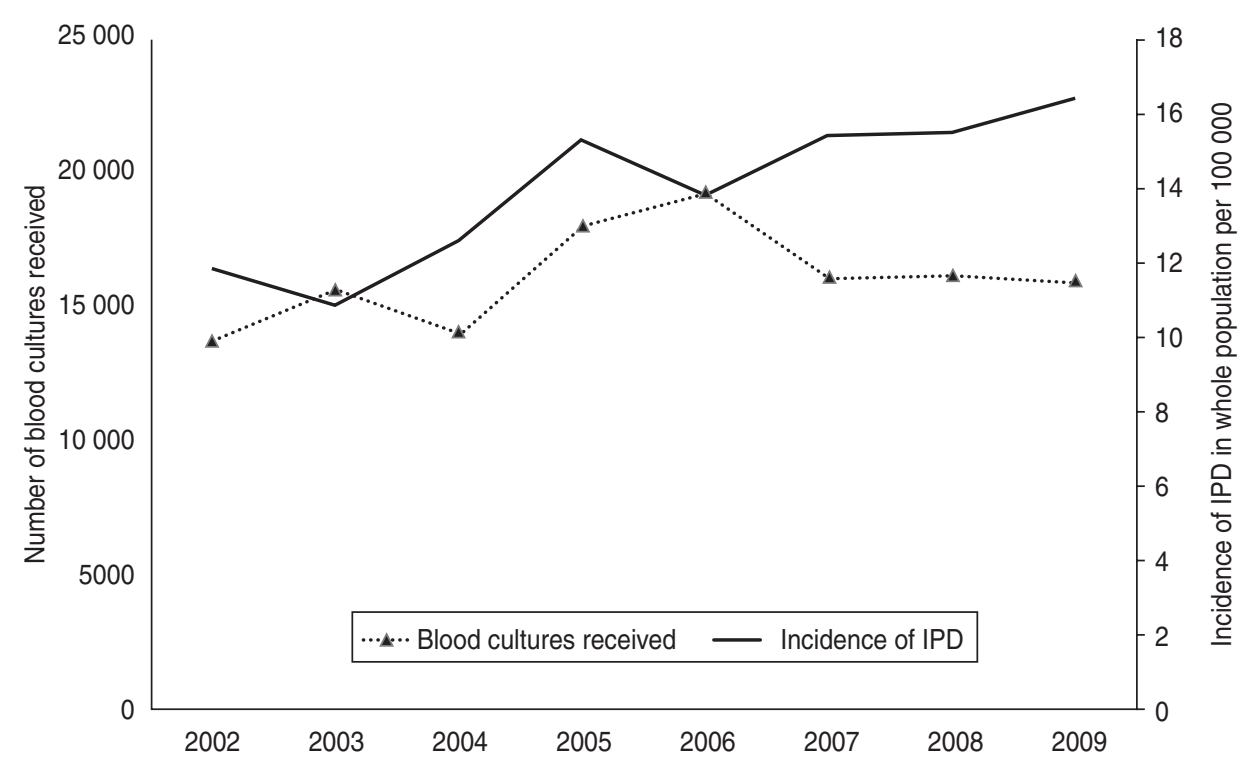

Fig. 4. Incidence of invasive pneumococcal disease (IPD) and number of blood culture samples received by Hull and East Yorkshire Hospitals NHS Trust.

compared to $48 \cdot 8(95 \%$ CI $41 \cdot 4-57 \cdot 2)$ per 100000 person-years in those without a record of vaccination prior to IPD; PPV23 effectiveness was thus estimated to be $15 \%(95 \% \mathrm{CI}-4 \cdot 6$ to 31$)$.

\section{Incidence of hospitalization with pneumonia}

The numbers and incidence of hospitalizations with pneumonia increased every year of the study period from 833 cases $(143 / 100000,95 \%$ CI $133-153)$ in 2002 to $1250(207 / 100000,95 \%$ CI $195 \cdot 5-218 \cdot 6)$ in 2009 (Poisson regression, $P<0 \cdot 001$ ), an increase in incidence of $45 \%$. The incidence of pneumonia by age range is shown in Figure 5. Incidence increased significantly in adults, with an increase of $44 \%$ in those aged $\geqslant 65$ years [from $543(95 \%$ CI 498-590) to 781 $(95 \%$ CI 729,836$)$ per 100000 (Poisson regression, $P<0 \cdot 001)$ ] and $72 \%$ in those aged $15-64$ years [from 
Table 2. Cases of invasive pneumococcal disease by pneumococcal serotype

\begin{tabular}{lllcc}
\hline \hline & $\begin{array}{l}\text { No. (\%) of } \\
\text { culture- positive } \\
\text { cases with } \\
\text { serotype result }\end{array}$ & $\begin{array}{l}\text { Serotypes } \\
\text { covered by } \\
\text { PPV and PCV* } \\
\text { (PCV7 serotypes) }\end{array}$ & $\begin{array}{l}\text { Serotypes } \\
\text { covered by } \\
\text { PPV but } \\
\text { not PCV } \dagger\end{array}$ & $\begin{array}{l}\text { Serotypes not } \\
\text { covered by PPV } \\
\text { or PCV } \\
\text { (non-vaccine serotypes) }\end{array}$ \\
\hline 2002 & $18(27 \cdot 7 \%)$ & $11(61 \cdot 1 \%)$ & $6(33 \cdot 3 \%)$ & $1(5 \cdot 6 \%)$ \\
2003 & $38(64 \cdot 4 \%)$ & $15(39 \cdot 5 \%)$ & $20(52 \cdot 6 \%)$ & $3(7 \cdot 9 \%)$ \\
2004 & $50(67 \cdot 6 \%)$ & $21(42 \cdot 0 \%)$ & $27(54 \cdot 0 \%)$ & $2(4 \cdot 0 \%)$ \\
2005 & $66(74 \cdot 2 \%)$ & $34(51 \cdot 5 \%)$ & $26(39 \cdot 4 \%)$ & $6(9 \cdot 1 \%)$ \\
2006 & $68(85 \cdot 0 \%)$ & $26(38 \cdot 2 \%)$ & $36(52 \cdot 9 \%)$ & $6(8 \cdot 8 \%)$ \\
2007 & $76(88 \cdot 4 \%)$ & $24(31 \cdot 6 \%)$ & $42(55 \cdot 3 \%)$ & $10(13 \cdot 2 \%)$ \\
2008 & $81(89 \cdot 0 \%)$ & $16(19 \cdot 8 \%)$ & $57(70 \cdot 4 \%)$ & $8(9 \cdot 9 \%)$ \\
2009 & $81(85 \cdot 3 \%)$ & $10(12 \cdot 3 \%)$ & $56(69 \cdot 1 \%)$ & $15(18 \cdot 5 \%)$ \\
\hline \hline
\end{tabular}

PPV, Pneumococcal polysaccharide vaccine; PCV, pneumococcal conjugate vaccine.

* Serotypes: 4, 6b, 9V, 14, 18c, 19f, 23f.

$\dagger$ Serotypes: 1, 2, 3, 5, 7f, 8, 9N, 10a, 11a, 12f, 15b, 17f, 19a, 20, 22f, 33f.

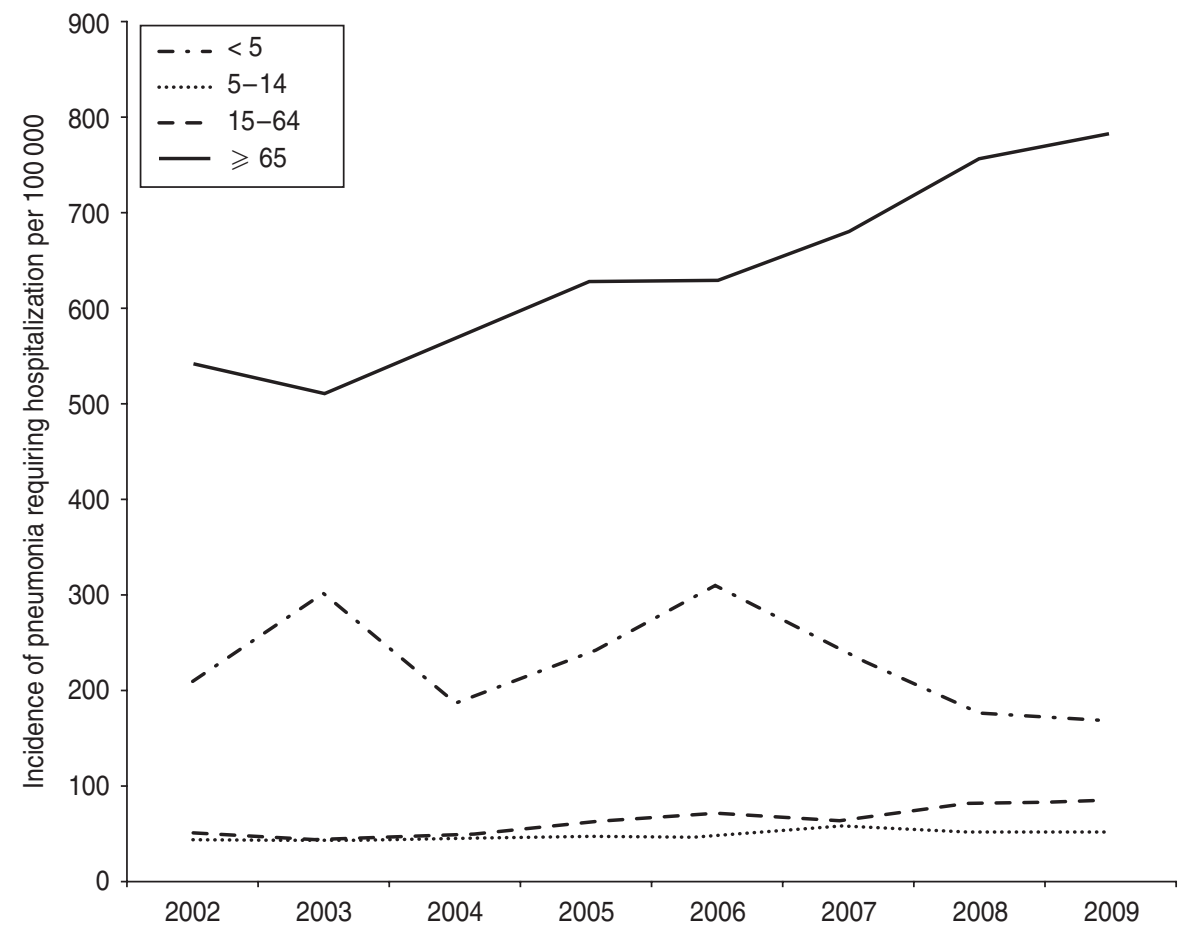

Fig. 5. Incidence of hospitalization with pneumonia by age range.

$48 \cdot 8(95 \%$ CI $42 \cdot 1-56 \cdot 4)$ to $84 \cdot 1(95 \%$ CI $75 \cdot 4-93 \cdot 6)$ per 100000 (Poisson regression, $P<0 \cdot 001$ )] from 2002 to 2009. Incidence in those aged $<5$ years did not change significantly between 2002 and 2005, but decreased in 2006-2009, from 309 (95\% CI 250-378) to 167 (95\% CI 126-218) (Poisson regression, $P<0 \cdot 001$ ) post-introduction of PCV7. Incidence in those aged 5-14 years did not change significantly over the study period (Poisson regression, $P=0 \cdot 90$ ).
Incidence of invasive group B meningococcal infection and of hospitalization with fractured neck of femur ('observational controls')

Reports of 98 invasive group B meningococcal infections were identified; the incidence of these infections was highly variable year to year and did not change significantly overall from 2002 to 2009 (Poisson regression, $P=0.78$ ) (Fig. 6). There were 5035 hospital 


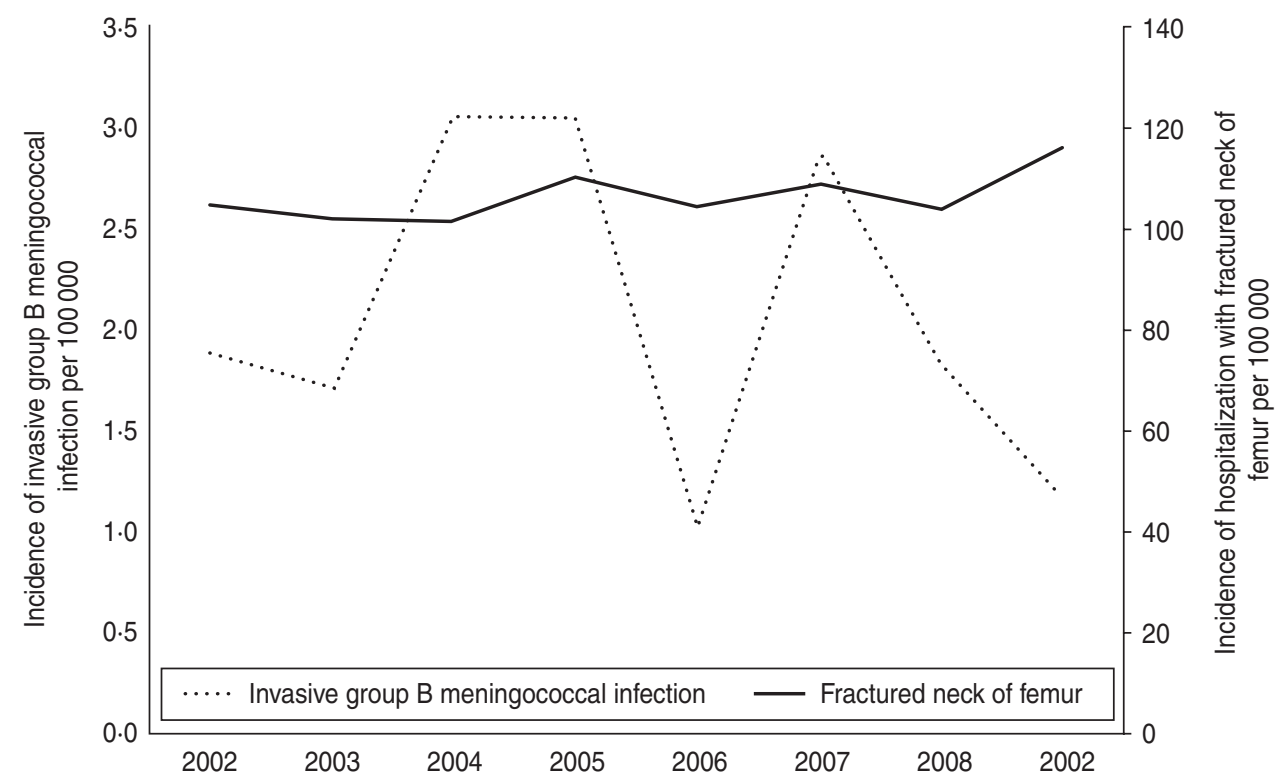

Fig. 6. Incidence of invasive group B meningococcal infection and of hospitalization with fractured neck of femur.

admissions with a primary diagnosis of fracture neck of femur over the study period and similarly there was no significant change in incidence (Poisson regression, $P=0 \cdot 22$ ) (Fig. 6).

\section{Geographical distribution of disease, vaccination uptake and deprivation indices}

Over the study period, the overall age-standardized incidence of IPD in Hull was $14 \cdot 3$ (95\% CI 12.8-16.0) per 100000 person-years and was considerably higher than that in ERoY, $8 \cdot 8(95 \%$ CI 7.7-9.9) per 100000 person-years. The incidence of IPD was significantly higher in Hull in the $\geqslant 65$ years (Poisson regression, $P<0 \cdot 001$ ) and the 15-64 years (Poisson regression, $P<0 \cdot 001)$ age groups, but not in children aged $<14$ years (Table 3). Similarly, the overall age-standardized incidence of hospitalization with pneumonia was higher in Hull (148.3, 95\% CI 143-153 per 100000 person-years) compared to ERoY (111.2, 95\% CI $107-115$ per 100000 person-years) over the study period. The incidence of pneumonia was significantly higher in Hull for all age groups except those aged $<5$ years (Table 3).

There was no significant difference in mean PPV23 uptake between Hull and ERoY over the study period [62.7\% (95\% CI 62.6-62.9) and 63.3\% (95\% CI $63 \cdot 2 \%, 63 \cdot 5 \%)$, respectively] $\left(\chi^{2}, P=0 \cdot 07\right)$. Mean uptake of PCV7 was marginally higher in ERoY from 2006 to 2009 [74.4\% (95\% CI 72.9-76.0) compared to $70.8 \%(95 \%$ CI $69.4 \%, 72 \cdot 3 \%)]\left(\chi^{2}, P=0.01\right)$, although it exceeded $94 \%$ in both areas by 2008 .
A higher incidence of IPD was noted in the most deprived Hull postcode district and incidence of IPD was significantly related to the postcode IMD score (Spearman's rank correlation, $R^{2}=0.62, P=0.04$ ) (Fig. 7). The same pattern was not noted in ERoY (data not shown), where there were less cases overall and a larger number of postcode districts.

There was no significant difference in case fatality within 30 days for IPD between Hull and ERoY [ $20 \%$ vs. $23 \%$, respectively $\left.\left(\chi^{2}, P=0 \cdot 26\right)\right]$, nor median age at death (which appeared lower in Hull at 78 years compared to 80.5 years in ERoY), (Mann-Whitney, $P=0 \cdot 40)$.

\section{DISCUSSION}

Enhanced surveillance of infection and vaccine uptake from 2002 has shown that despite increasing uptake of both vaccines, and contrary to our primary hypothesis, the overall incidence of IPD and pneumonia has increased. Importantly, the incidence rates for IPD presented are likely to be an underestimate of the true rates: many individuals will not present to hospitals (particularly the very elderly); diagnosis of IPD is dependent on appropriate samples being sent at the appropriate time (ideally blood cultures prior to antimicrobial therapy); and some residents of Hull and ERoY may have presented elsewhere for diagnosis and treatment. Whereas other UK studies have explained an observed increase in IPD incidence as an artefact of increased sampling [27, 28], our findings do 
Table 3. Incidence density of invasive pneumococcal disease (IPD) and pneumonia by age in Hull and East Riding of Yorkshire (ERoY), 2002-2009

\begin{tabular}{lcc}
\hline \hline Age range (years) & Hull & ERoY \\
\hline Incidence density of IPD per $\mathbf{1 0 0} 00$ person-years $\mathbf{( 9 5} \% \mathbf{C I})$ & \\
$\geqslant 65$ & $60 \cdot 6(52 \cdot 2-70 \cdot 1)$ & $34 \cdot 0(29 \cdot 0-39 \cdot 6)$ \\
$15-64$ & $9 \cdot 1(7 \cdot 6-10 \cdot 9)$ & $5 \cdot 4(4 \cdot 3-6 \cdot 6)$ \\
$5-14$ & $3 \cdot 1(1 \cdot 3-6 \cdot 1)$ & $2 \cdot 1(0 \cdot 8-4 \cdot 5)$ \\
$<5$ & $16 \cdot 3(10 \cdot 1-25 \cdot 0)$ & $21 \cdot 2(13 \cdot 9-31 \cdot 1)$ \\
Incidence density of pneumonia per $\mathbf{1 0 0 0 0 0}$ person-years $\mathbf{( 9 5 \% \mathbf { C I } )}$ \\
$\geqslant 65$ & $737(707-769)$ & $585(564-606)$ \\
$15-64$ & $72 \cdot 3(67 \cdot 9-76 \cdot 9)$ & $56 \cdot 0(52 \cdot 5-59 \cdot 7)$ \\
$5-14$ & $60 \cdot 1(51 \cdot 0-71 \cdot 3)$ & $37 \cdot 9(31 \cdot 2-45 \cdot 6)$ \\
$<5$ & $248(222-277)$ & $207(183-234)$ \\
\hline \hline
\end{tabular}

CI, Confidence interval.

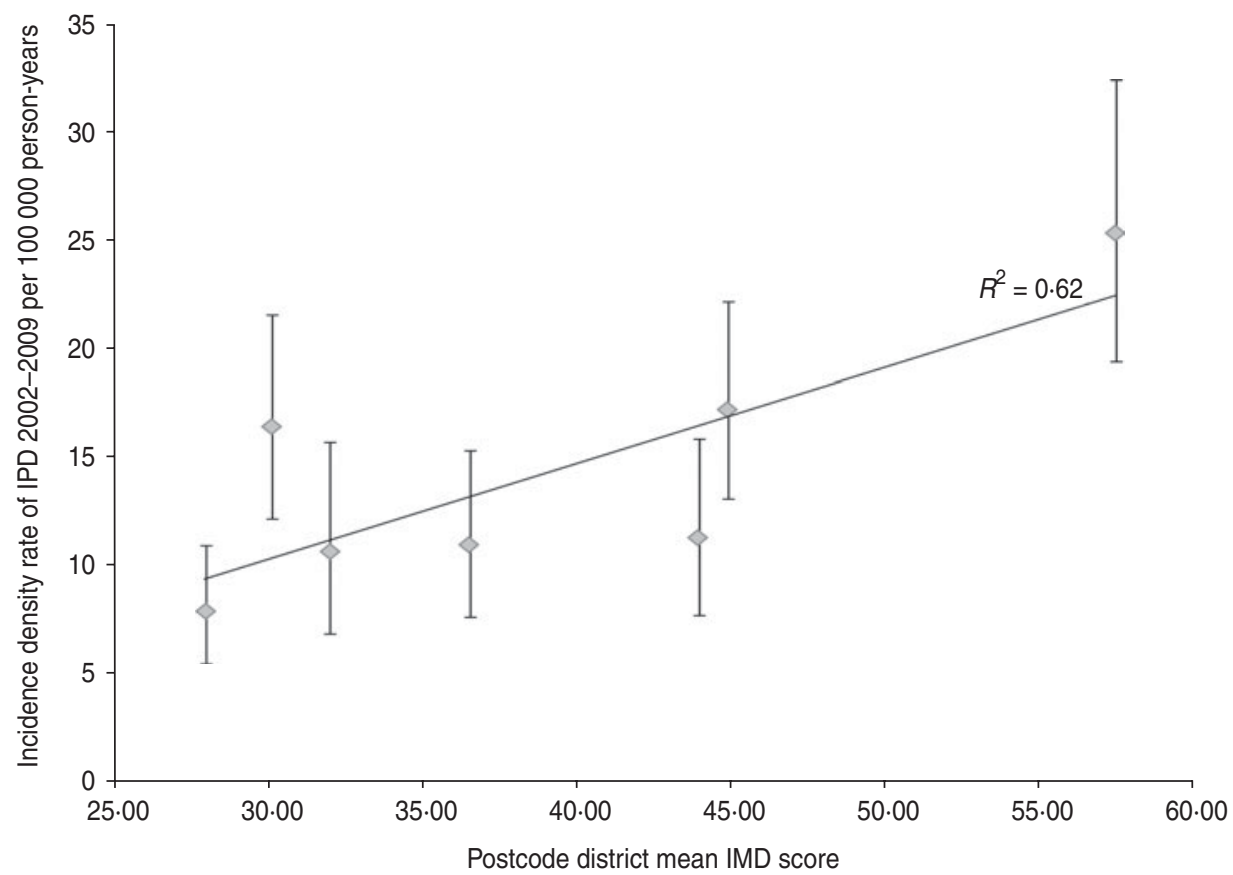

Fig. 7. Age-standardized incidence density rate of invasive pneumococcal disease (IPD) in Hull 2002-2009 by postcode district* and postcode index of multiple deprivation (IMD) score. [ ${ }^{*}$ Each point represents one postcode district in Hull with population > 20000 (HU3-9). HU1 and HU2 were excluded as the population of both is $<4000$.]

not support this as the number of blood cultures performed remained relatively stable during the study period (and the incidence of blood culture sampling appeared to decrease). The dramatic increase in the incidence of hospitalization for pneumonia, in keeping with a recent large epidemiological study in England and Wales, also suggests that the burden of pneumococcal disease is increasing in this locality [29].

A reduction in IPD was not demonstrated in the main target group for PPV23 or overall. The protective effect of PPV23 at the population level was found to be low (and may be overestimated due to underreporting of PPV23 vaccination status), and is consistent with the recent assessment by the Joint Committee on Vaccination and Immunization (JCVI) UK, that the effectiveness of PPV23 is poor in those aged $\geqslant 65$ years [30]. At the patient level, many 'vaccine failures' were identified. However, the increasing incidence of IPD may be unrelated to vaccination; for example, risk factors for IPD may have increased in adults during the study period. In contrast, we found 
a significant reduction in the incidence of pneumonia in children aged $<5$ years following the introduction of PCV7. While the reduction in the incidence of IPD in children was not statistically significant, we acknowledge that the number of IPD cases in children was small and the study may therefore have been performed in too small a population area to detect the anticipated target population effects of PCV7 seen in other studies [11-13] [the population aged $<5$ years was $32900(5 \cdot 4 \%$ of the total population) in 2009] [19]. However, there was no evidence of herd immunity in the larger older cohort during the post-PCV (post-2006) period. Whether this is due to a delayed beneficial effect yet to be detected, the relatively small size of our study compared to some reports, natural fluctuation of disease incidence, or another reason is unclear.

The incidence rates of IPD in Hull and ERoY are higher than those reported nationally and are considerably higher than those in Oxfordshire. National surveillance requires voluntary reporting of clinically significant isolates by individual microbiology laboratories whereas in this study, information on relevant isolates was actively sought. Therefore, the higher incidence rates for Hull and ERoY may reflect the more inclusive methodology. However, this does not explain the large difference in rates between Hull and ERoY and Oxfordshire, where enhanced surveillance was also performed, and suggests a genuinely higher burden of IPD in our locality compared to more affluent and healthier areas of the UK. It is also notable that rates of IPD are falling in Oxfordshire. Together with the observed marked differences in the incidence of both IPD and hospitalization for pneumonia between Hull and ERoY, this study highlights the likely presence of important health inequalities within the population of the UK. Interestingly, HEY Hospitals NHS trust was recently identified by the Dr Foster group as having a higher mortality rate compared to the average acute UK hospital and it is suspected that this, at least in part, is explained by the recognized high level of comorbidity and risk-taking behaviour in the local population [31]. The higher incidence of IPD in the most deprived Hull postcode district, the apparent relationship between the incidence of IPD and postcode IMD score within Hull postcode districts, and the differences in the incidences of IPD and pneumonia between Hull and the more affluent ERoY, also suggests that social deprivation is an important contributor to IPD, in keeping with a previous study of children [32]. Deprivation covers a broad range of issues and refers to unmet needs resulting from a lack of resources of all kinds, not just financial [25]. The IMD score reflects the measurement of seven distinct domains of deprivation (differentially weighted and combined) which include: income deprivation, employment deprivation, health deprivation and disability, education skills and training deprivation, barriers to housing and services, living environment deprivation, and crime [25]. In every domain the population of Hull is considered more deprived than the average for England, and even within Hull there are also marked inequalities [20, 25]. It is conceivable that deprivation affecting each of the seven domains might contribute to increased pneumococcal carriage and disease (e.g. it can be speculated that overcrowded living conditions might lead to increased transmission and carriage of pneumococcus; and poverty, disability and low educational level might contribute to delayed presentation of infection and increased likelihood of invasive disease). However, further research (at the individual level) is indicated to confirm that such a relationship exists and to identify the important underlying social determinants.

The increased burden of pneumococcal disease observed in this study is not explained easily by changes in population demographics; although the numbers of elderly did increase, the growth in the population aged $\geqslant 65$ years $(4 \cdot 6 \%)$ was modest over the 8 -year period and similar to the increase $(3.5 \%)$ in the general population. The proportion of the population aged $>80$ years was unchanged $(4.3 \%$ in $2002,4.6 \%$ in 2009) [19]. Although ERoY has an older population compared to Hull and England, the population of Hull has a lower proportion of elderly, thus the combined population structure of Hull and ERoY is similar to that of England [19]. The increases in the incidence of IPD and pneumonia overall were largely driven by increases in the adult age groups. The significant increase in both IPD and pneumonia in the 15-64 years age group could be explained by an increasing prevalence of risk factors for pneumococcal disease known to be at a high level in the young adult population of Hull, such as intravenous drug use, high alcohol intake, and smoking [20, 25], although further research is required to confirm this; such factors should be important targets for future local health promotion and research. The increase is not explained by antibiotic resistance. We were not able to assess if the prevalence of pneumococcal carriage in the population also increased. There were no major 
changes in laboratory equipment or blood culture methodology and, while PCR was used more frequently in later years, the number of cases diagnosed by this method was low (14 cases, $2 \%$ ) spread over the study period (1/59 cases in 2003 vs. 4/95 cases in 2009). Uptake of both PPV23 and PCV7 was higher in Hull and ERoY compared to the averages for England [22, 33], and although there was marginally higher uptake of PCV7 in ERoY compared to Hull, PCV7 uptake in both areas was high following the first full year of vaccine implementation; PPV23 uptake was similar in both areas. Thus our observations cannot be attributed to low vaccine coverage and the marked differences between Hull and ERoY are unlikely to be explained by differential vaccine uptake.

The reduction in PCV7 serotypes noted after 2006 is consistent with the observations of other studies and supports the effectiveness of this vaccine in reducing vaccine serotype disease. However, although an increase in non-PCV7 serotypes after 2006 was to be expected and in keeping with other studies, the observed overall increase in the incidence of IPD was surprising and in contrast to other settings where IPD rates have been falling post-PCV7 [14-17]. It is therefore conceivable that a change in the seroepidemiology of IPD in our locality, in the period following PCV7 implementation, has contributed to an increased overall burden of disease; this appears to have mostly been due to an increase in non-PCV7, PPV23 serotypes. A recent large cohort study showed that some pneumococcal serotypes result in higher mortality (and presumably therefore more severe, non-fatal illness) compared to others [34]. Those serotypes covered by PCV7 are associated with relatively lower 30-day mortality compared to many non-PCV, PPV serotypes and non-vaccine serotypes [34]. One consequence of PCV7 introduction in our locality, therefore, may have been replacement of PCV7 serotypes with serotypes more likely to cause severe disease, although there was no clear evidence of a significant increase in mortality, which was $19 \%$ in the 2002-2005 period compared to $24 \%$ in the 2006-2009 period $\left(\chi^{2}, P=0 \cdot 20\right)$. Differences in the predisposition of serotypes to colonize and invade the human respiratory tract might have also contributed to the overall increase in IPD incidence [35]. The notable increase in mortality (from $22 \%$ to $37 \%$ ) between 30 days and 1 year after the diagnosis of IPD entirely occurred in adults ( $>16$ years), especially those aged $\geqslant 65$ years, and suggests that IPD is a marker of poor 1-year survival. This is likely to be due to comorbidity, but other explanations, such as the triggering of an inflammatory response resulting in cardiovascular disease should be investigated.

Our results suggest that the current approach to pneumococcal vaccination in our locality should be reviewed. Possible alternative strategies include a switch to the use of conjugate vaccines in those aged $\geqslant 65$ years (and high-risk patients of any age) [36], and the development and use of conjugate vaccines that cover a higher proportion of circulating serotypes. Studies are currently being performed to assess the effectiveness of conjugate compared to polysaccharide vaccines in older age groups [37], but it remains unclear as to whether this will be a more costeffective strategy than PPV23. The UK has recently switched to PCV13 for the vaccination of those aged $<2$ years and it will be interesting to observe the impact of this in our locality on IPD and pneumonia both within this age group and in the overall population. The results also highlight the importance of tackling the likely underlying public health determinants of IPD and pneumonia.

We acknowledge that this study has a number of limitations. The observational design means that the observed trends cannot be definitively attributed to vaccine effectiveness and could be due to natural fluctuation. It is, however, interesting to note that the incidence of invasive meningococcal group B infections did not increase over the study period. The estimate of vaccination uptake was based on data from primary-care practitioners both at a local and national level. In contrast to other areas in England, a financial incentive is offered in Hull and ERoY by the HPU for information on PPV23 uptake. The vaccination surveillance database is updated continuously, with active measures taken annually to identify and contact non-responding GP practices. Nevertheless, PPV23 uptake is likely to be underestimated. There are also limitations to assessing deprivation by postcode districts, which may contain both areas of deprivation and comparative affluence. Use of the IMD score, which is calculated relative to populations in other postcode districts within the same region, presents some challenges for interpretation when the whole region is relatively deprived (as in Hull). In addition, there are recognized limitations in using population estimates for the postcode districts derived from primary care (GP) registrations (PCTs utilize this data in the absence of ONS population estimates at this geographical level) which tend to overestimate population size compared to ONS estimates 
[38]; incidence density rates of IPD in the postcode districts might be therefore be underestimated, and if there was differential registration between populations of different postcode districts consistently over the 8-year study period then the association between deprivation and IPD by postcode district, might be either over- or under-estimated. We tried to account for differences in age distribution within postcode districts by using directly age-standardized data. The identification of those hospitalized with pneumonia relied on ICD-10 based case-coding performed after hospital discharge. While a change in coding practice and/or clinical practice (e.g. lower threshold for hospital admission) may have occurred over the study period, this is unlikely to have resulted in the magnitude of the observed increases in pneumonia incidence. Indeed the relatively constant incidence of the observation control, i.e. hospitalization with fractured neck of femur, would suggest that there was not a systematic change in coding practice or other gross bias in data collection over the study period and thus provides supportive evidence of a true increase in pneumonia incidence. There are many causes o pneumonia and the increase cannot be solely attributed to an increase in the incidence of $S$. pneumoniae infection, although $S$. pneumoniae is well recognized to be the commonest cause of CAP worldwide. We included only patients given a primary (first position) diagnostic code for pneumonia in order to increase the likelihood of identifying true cases of CAP. Finally, the proportion of isolates sent to the reference laboratory for typing was particularly low in the first year (2002), but was higher thereafter (between $64 \%$ and $89 \%$ ); this could have affected our results as it is possible, for example, that isolates from patients with more severe pneumococcal infection are more likely to be sent for serotyping. Despite the identified limitations, this study provides valuable information on the epidemiology of IPD and pneumonia and the early effects of vaccination in a relatively deprived UK population and suggests that the impact of vaccination cannot always be assumed to be consistent across populations.

In conclusion, the burden of IPD and pneumonia increased in Hull and ERoY from 2002 to 2009 despite improved uptake of both PPV23 and PCV7 vaccines. The underlying determinants of this trend are unclear, and may be independent of vaccine introduction, but should be explored. Existing vaccination programmes alone might not be sufficient to reverse the burden of pneumococcal disease, particularly in areas with a high prevalence of comorbid illness and social deprivation, and our findings are consistent with the JCVI assessment that PPV23 effectiveness is poor in those aged $\geqslant 65$ years [28]. Further investigation of the social determinants of pneumococcal disease is required. Surveillance of pneumococcal serotype distribution and monitoring of non-PCV serotypeassociated disease is imperative to inform future PCV formulations. Awareness of pneumococcal disease by healthcare professionals remains of paramount importance.

\section{NOTE}

Supplementary material accompanies this paper on the Journal's website (http://journals.cambridge.org/ hyg).

\section{ACKNOWLEDGEMENTS}

The hospitals involved in the surveillance were as follows: Hull Royal Infirmary, Castle Hill, Princess Royal, York, Scunthorpe General, Scarborough District General, Bridlington and Goole and District. East Riding of Yorkshire and Hull Primary Care Trusts provided population data. The authors acknowledge the help of Alexander Anderson, Peter Cowling, Dan Weiand and Naomi Thompson for their help in providing and interpreting the hospital data; Emma Smith, Paul Thomas, Jeremy Newton and Helen Fields for their help in providing and interpreting data from the Healthcare Purchaser System Database; Mandy Porter and Adrian Wensley for help with statistical analyses; and Tracey Docwra and Pawel Wlodarczyk for technical assistance.

\section{DECLARATION OF INTEREST}

None.

\section{REFERENCES}

1. World Health Organization. Pneumococcal conjugate vaccine for childhood immunisation-WHO position paper. Weekly Epidemiological Record 2007; 82: 93-104.

2. Melegaro A, et al. The current burden of pneumococcal disease in England and Wales. Journal of Infection 2006; 52: 37-48.

3. Fine MJ, et al. Efficacy of pneumococcal vaccination in adults. A meta-analysis of randomized controlled trials. Archives of Internal Medicine 1994; 154: 2666-2677. 
4. Melegaro A, Edmunds WJ. The 23-valent pneumococcal polysaccharide vaccine. Part I. Efficacy of PPV in the elderly: a comparison of meta-analyses. European Journal of Epidemiology 2004; 19: 353-363.

5. Moberley SA, et al. Vaccines for preventing pneumococcal infection in adults. Cochrane Database of Systematic Reviews 2008; Issue 1, Art. No. CD000422.

6. Huss A, et al. Efficacy of pneumococcal vaccination in adults: a meta-analysis. Canadian Medical Association Journal 2009;180: 48-58.

7. Maruyama T, et al. Efficacy of 23-valent pneumococcal vaccine in preventing pneumonia and improving survival in nursing home residents: double blind, randomised and placebo controlled trial. British Medical Journal 2010; 340 : c1004.

8. Jackson LA, et al. Effectiveness of pneumococcal polysaccharide vaccine in older adults. New England Journal of Medicine 2003; 348: 1747-1755.

9. Shapiro ED, et al. The protective efficacy of polyvalent pneumococcal polysaccharide vaccine. New England Journal of Medicine 1991; 325: 1453-1460.

10. Foster D, et al. Invasive pneumococcal disease: epidemiology in children and adults prior to implementation of the conjugate vaccine in the Oxfordshire region, England. Journal of Medical Microbiology 2008; 57: 480-487.

11. Centers for Disease Control and Prevention. Invasive pneumococcal disease in children 5 years after conjugate vaccine introduction-eight states, 1998-2005. Morbidity and Mortality Weekly Report 2008; 57: 144-148.

12. Lexau CA, et al. Changing epidemiology of invasive pneumococcal disease among older adults in the era of pediatric pneumococcal conjugate vaccine. Journal of the American Medical Association 2005; 294: 20432051.

13. Whitney CG, et al. Decline in invasive pneumococcal disease after the introduction of protein-polysaccharide conjugate vaccine. New England Journal Medicine 2003; 348: 1737-1746.

14. Rodenburg GD, et al. Effects of pneumococcal conjugate vaccine 2 years after its introduction, the Netherlands. Emerging Infectious Diseases 2010; 16: 816-823.

15. Health Protection Agency. Cumulative weekly number of reports of invasive pneumococcal disease due to any of the seven serotypes in Prevenar $^{\mathrm{TM}}$ : children aged $<2$ years in England and Wales by epidemiological year: July-June (2003-to date) (http://www.hpa.org.uk/web/ HPAweb\&HPAwebStandard/HPAweb_C/1207821645727). Accessed December 2010.

16. Health Protection Agency. Cumulative weekly number of reports of invasive pneumococcal disease due to any of the serotypes NOT in Prevenar ${ }^{\mathrm{TM}}$ : children aged $<2$ years in England and Wales by epidemiological year: July-June (2003-to date) (http://www.hpa.org.uk/web/ HPAweb\&HPAwebStandard/HPAweb_C/1207821646480). Accessed December 2010.

17. Foster D, et al. Reduction in invasive pneumococcal disease following implementation of the conjugate vaccine in the Oxfordshire region, England. Journal of Medical Microbiology 2011; 60: 91-97.

18. Hausdorff WP, et al. Which pneumococcal serogroups cause the most invasive disease: implications for conjugate vaccine formulation and use, part 1. Clinical Infectious Diseases 2000; 30: 100-121.

19. Office for National Statistics. 2006-based sub national population projections (http://www.statistics.gov.uk/ downloads/theme_population/SNPP-2006/Table2.xls). Accessed August 2010.

20. APHO and Department of Health. Health profile 2010, Kingston upon Hull. (C Crown Copyright 2010.)

21. APHO and Department of Health. Health profile 2010, East Riding of Yorkshire. (C Crown Copyright 2010.)

22. Health Protection Agency. Pneumococcal vaccination uptake monitoring on behalf of Department of Health (http://www.hpa.org.uk/Topics/InfectiousDiseases/ InfectionsAZ/Pneumococcal/PPVUptake/\#03/04). Accessed October 2010.

23. Orenstein WA, et al. Field evaluation of vaccine efficacy. Bulletin of the World Health Organisation 1985; 63: $1055-1068$.

24. Association of Public Health Observatories. Commonly used public health statistics and their confidence intervals (http://www.apho.org.uk/resource/item.aspx?RID = 48457). Accessed 29 June 2011.

25. Department for Communities and Local Government. The English Indices of Deprivation 2007, Summary. (C) Crown Copyright 2007.)

26. Perneger TV. What's wrong with Bonferroni adjustments. British Medical Journal 1998; 316: 12361238.

27. Trotter CL, et al. Epidemiology of invasive pneumococcal disease in the pre-conjugate vaccine era: England and Wales, 1996-2006. Journal of Infection 2009; 60: 200-208.

28. Ihekweazu CA, et al. Trends in incidence of pneumococcal disease before introduction of conjugate vaccine: South West England, 1996-2005. Epidemiology and Infection 2008; 136: 1096-1102.

29. Trotter CL, et al. Increasing hospital admissions for pneumonia, England. Emerging Infectious Diseases $2005 ; \mathbf{1 4} ; 727-733$.

30. Department of Health. JCVI statement on discontinuation of the routine pneumococcal vaccination programme for adults aged 65 years and older, 16 March 2011 (http://www.dh.gov.uk/ab/JCVI/DH_094744). Accessed 16 March 2011.

31. Dr Foster Health. Hull and East Yorkshire Hospitals NHS Trust-quality report 2009/10 (http://www. drfosterhealth.co.uk/quality-reports/trust.aspx?otype = 2\&id=271). Accessed 13 March 2011.

32. Grant CC, et al. Invasive pneumococcal disease in Oxford, 1985-2001: a retrospective case series. Archives of Disease in Childhood 2003; 88: 712-714.

33. The Health and Social Care Information Centre. NHS Immunisation Statistics, England (http://www.ic.nhs. $\mathrm{uk} /$ statistics-and-data-collections/health-and-lifestyles/ immunisation/nhs-immunisation-statistics-england2009-10). Accessed 7 July 2011. 
34. Harboe ZB, et al. Pneumococcal serotypes and mortality following invasive pneumococcal disease: a population-based cohort study. PLoS Medicine 2009; 6: e1000081.

35. Hausdorff WP, Feikin DR, Klugman KP. Epidemiological differences among pneumococcal serotypes. Lancet Infectious Diseases 2005; 5: 83-93.

36. Dransfield MT, et al. Superior immune response to protein-conjugate versus free pneumococcal polysaccharide vaccine in chronic obstructive pulmonary disease. American Journal of Respiratory and Critical Care Medicine 2009; 180: 499-505.

37. Goldblatt D, et al. The immunogenicity of 7-valent pneumococcal conjugate vaccine versus 23 -valent polysaccharide vaccine in adults aged 50-80 years. Clinical Infectious Diseases 2009; 49: 1318-1325.

38. The Health and Social Care Information Centre. GP registered populations (http://www.ic.nhs.uk/statisticsand-data-collections/population-and-geography/gpregistered-populations). Accessed 12 July 2011. 